\title{
Floquet stability of discrete breathers in anisotropic Josephson junction ladders
}

\author{
P.J. Martínez ${ }^{\text {a,1 }}$, L.M. Floría b,*, J.L. Marín b,c ${ }^{\text {b. S. Aubry d, J.J. Mazo }}{ }^{\text {b,e }}$ \\ a Departamento de Física Aplicada, Instituto de Ciencia de Materiales de Aragón, Universidad de Zaragoza, 50009 Zaragoza, Spain \\ ${ }^{\mathrm{b}}$ Departamento de Física de la Materia Condensada, Instituto de Ciencia de Materiales de Aragón, \\ Universidad de Zaragoza, 50009 Zaragoza, Spain \\ ${ }^{\mathrm{c}}$ Department of Applied Mathematics and Theoretical Physics, University of Cambridge, CB3 9EW Cambridge, UK \\ d Laboratoire Léon Brillouin (CEA-CNRS), CE Saclay, 91191 Gif-sur-Yvette Cedex, France \\ e Department of Electrical Engineering and Computer Science, Massachusetts Institute of Technology, 02139 Cambridge, MA, USA
}

Received 28 May 1997; received in revised form 17 December 1997

\begin{abstract}
We report on preliminary numerical results on linear stability (Floquet) analysis of discrete breather solutions of the resistively and capacitively shunted junction (RCSJ) dynamics of an anisotropic ladder of Josephson junctions biased by time periodic, uniform currents. Different types of bifurcations, driven by exponentially localized eigenvectors of the monodromy matrix, are shown to destabilize the intrinsic localized modes, when parameters such as Josephson coupling, resistive coupling or external currents intensity are varied. We show some two-dimensional sections of the computed sector of the stability phase diagram in the corresponding parameter space. (c) 1998 Elsevier Science B.V.
\end{abstract}

\section{Introduction}

Recent theoretical analyses of the dynamics of an anisotropic Josephson junction ladder with injected AC currents [1,2] have shown the existence of intrinsic localized modes (discrete breathers) as attracting solutions of the equations of motion describing the dynamics of the system in the framework of the resistively and capacitively shunted junction (RCSJ) approach [3]. Since nowadays one can have an excellent control over the parameters of the fabricated arrays of Josephson junctions, these devices offer the opportunity of performing detailed experimental studies on nonlinear localization, where not only theoretical predictions can be adequately checked, but also new questions on discrete breathers challenging our present comprehension [4] could eventually arise.

The existence of discrete breathers in Josephson junction arrays should indeed be regarded as generic, given the connection between the general description of these systems in terms of the superconducting Ginzburg-Landau

\footnotetext{
* Corresponding author. E-mail: pedro@superpedro.unizar.es.

${ }^{1}$ E-mail: floria@posta.unizar.es.
} 
order parameter $\Psi(x)=|\Psi(x)| \mathrm{e}^{\mathrm{i} \theta(x)}$ (where $x$ denotes the island position), and the discrete nonlinear Schrödinger equation, for the case of ideal (perfect insulating) junctions [2]. In fact, the quantum Hamiltonian of a single ideal Josephson junction corresponds to the problem of two coupled anharmonic quantum oscillators, for which the asymmetric classical breather solutions have been shown to persist in the quantum regime as very long lifetime states [5]. When the energy cost to add an extra Cooper pair on a neutral superconducting island (charging energy $E_{\mathrm{c}}$ ) is much lower than the tunneling energy (Josephson energy $E_{\mathrm{J}}$ ), the superconducting phase $\theta(x)$ becomes a good (very weakly fluctuating) variable to describe the island state, thus validating the RCSJ approach [3]. This is the case when the superconducting islands are of macroscopic size. The validity of the RCSJ approach in the regime $E_{\mathrm{c}} / E_{\mathrm{J}} \ll 1$ is a well-established issue; see, for example, the excellent quantitative agreement of the RCSJ-based predictions and experimental results on the (DC) $I-V$ characteristics for annular arrays of Josephson junctions, recently reported by Watanabe et al. [6].

Here we will report on numerical results concerning the linear stability (Floquet) analysis of discrete breathers in anisotropic Josephson junction ladders (see Fig. 1) within the framework of the RCSJ approach. $\theta_{i}$ and $\theta_{i}^{\prime}$ will denote, respectively, the phases of upper and lower islands at site $i$ in the ladder; the currents $I(t)=I_{\mathrm{AC}} \cos (\omega t)$ are injected into the islands in the upper row and extracted from those in the lower row; $\left(J_{x}, \epsilon_{x}\right)$ are the junction characteristics for junctions in horizontal links and $\left(J_{y}, \epsilon_{y}\right)$ for junctions in vertical links. With the change of variables $\chi_{i}=\frac{1}{2}\left(\theta_{i}+\theta_{i}^{\prime}\right), \phi_{i}=\frac{1}{2}\left(\theta_{i}-\theta_{i}^{\prime}\right)$, the RCSJ equations [1,2] become

$$
\begin{aligned}
\ddot{\chi}_{i}= & J_{x}\left[\sin \left(\chi_{i+1}-\chi_{i}\right) \cos \left(\phi_{i+1}-\phi_{i}\right)+\sin \left(\chi_{i-1}-\chi_{i}\right) \cos \left(\phi_{i-1}-\phi_{i}\right)\right]+\epsilon_{x}\left(\dot{\chi}_{i+1}+\dot{\chi}_{i-1}-2 \dot{\chi}_{i}\right), \\
\ddot{\phi}_{i}= & J_{x}\left[\cos \left(\chi_{i+1}-\chi_{i}\right) \sin \left(\phi_{i+1}-\phi_{i}\right)+\cos \left(\chi_{i-1}-\chi_{i}\right) \sin \left(\phi_{i-1}-\phi_{i}\right)\right] \\
& +\epsilon_{x}\left(\dot{\phi}_{i+1}+\dot{\phi}_{i-1}-2 \dot{\phi}_{i}\right)-J_{y} \sin \left(2 \phi_{i}\right)-2 \epsilon_{y} \dot{\phi}_{i}-I(t) .
\end{aligned}
$$

With uniform initial conditions in the "center of mass" coordinates and momenta, i.e. $\chi_{i}$ and $\dot{\chi}_{i}$ independent of $i$, Eqs. (1) have the solution $\chi_{i}(t)=\Omega t+\alpha$ for all $i$. This effectively decouples Eqs. (2) for the $\phi_{i}$ variables from Eqs. (1) for the $\chi_{i}$ variables. Then, using efficient continuation methods [7,8] from the uncoupled limit $\left(J_{x}=\epsilon_{x}=0\right)$, one easily computes discrete breather solutions. These turn out to be attractors of the dynamics of the ladder in a wide range of parameter values (see, for example, [9]).

We will concentrate here on the rotobreather type of solutions, in which the phase half-difference $\phi_{j^{*}}$ trough a vertical junction rotates, while the rest $\phi_{i}\left(i \neq j^{*}\right)$ oscillate, and the "center of mass" variables $\chi_{i}$ remain uniformly at rest ( $\Omega=\alpha=0$; note that any other values for these parameters, fixed by the uniform initial conditions, would show the same behavior). The period of the rotobreather solution is $T_{\mathrm{b}}=2 \pi / \omega_{\mathrm{b}}=4 \pi / \omega$, where $\omega$ is the frequency of the external currents. Two characteristic features make this rotobreather solution particularly interesting. First, the voltage through the vertical link at $j^{*}$, where localization occurs, has non-zero mean value, so that a direct measure of the mean voltage profile will reveal the presence of the intrinsic localized mode in a feasible experiment, provided the device is adequately designed. Second, the excitation energy of this mode (which is a microscopic quantity due to its exponentially localized character) manifests itself through the intermittent creation and subsequent annihilation of a pair of fluxoid quanta of opposite sign (vortex-antivortex pair) located in the central plaquettes (Fig. 1(b)).

Rotobreathers were numerically found for the first time by Takeno and Peyrard [10], for a one-dimensional Hamiltonian array of pendulums with periodic coupling. These authors have later worked out approximate analytical expressions for them [11]. Their existence proof was implicitly envisaged in the general theorems of Mackay and Aubry [7], and explicitly considered by Aubry [8,12]. Numerical computations of rotobreathers in Josephson junction ladders have been discussed by Floría et al. [1,2]. Recently, for Eqs. (2) with uniform $\chi_{i}$ and constant (DC) current $I$, Mackay and Sepulchre [9] have shown the attracting character of rotobreather solutions. Though at present there are no experiments on Josephson junction ladders concerning these theoretical predictions, some earlier experiments on two-dimensional Josephson junction arrays [13] are indeed suggestive of tempting interpretations 

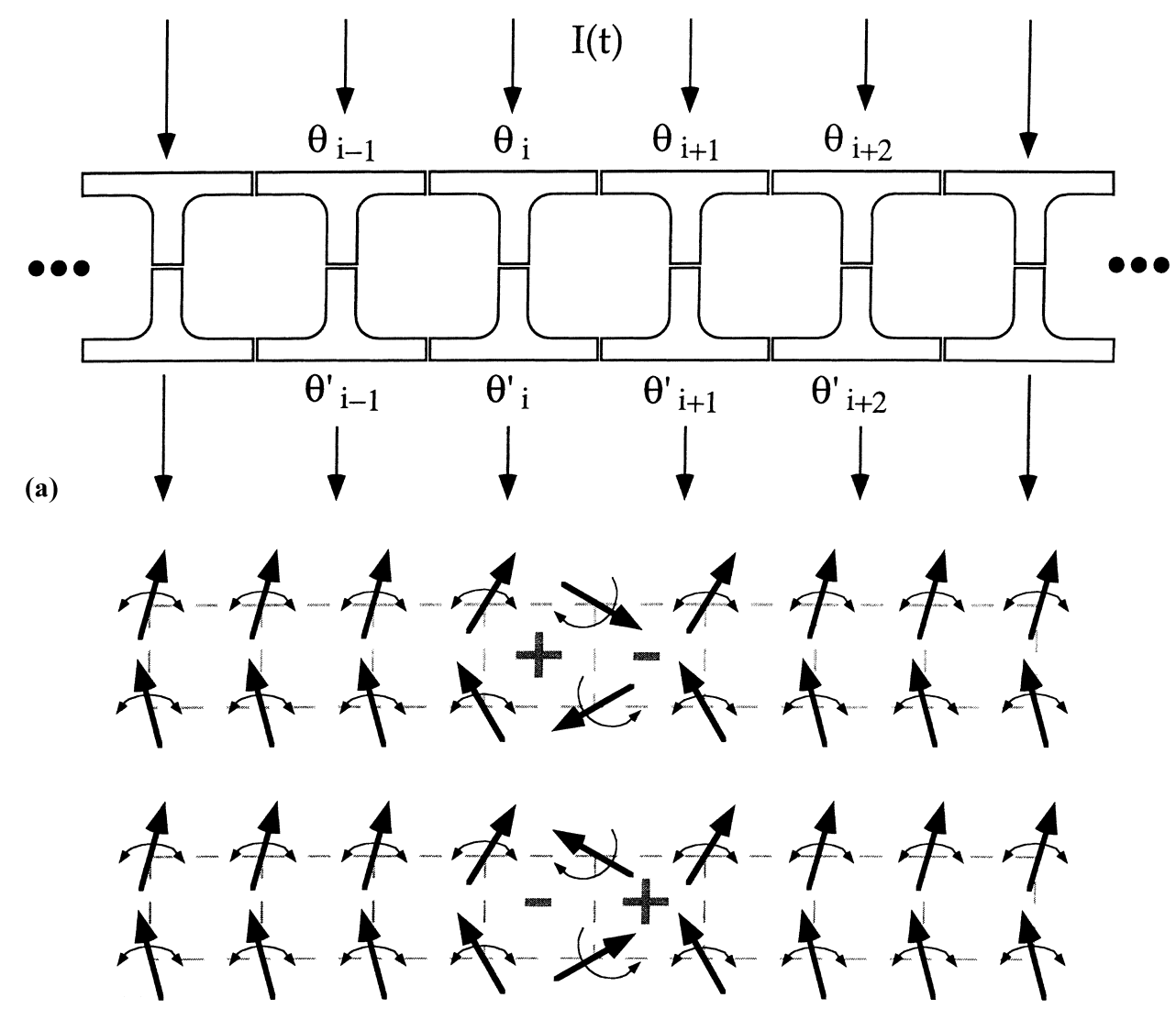

(b)

Fig. 1. Schematic picture of the JJ ladder showing the injection of the currents in the array (a), and the superconducting phase motion for the rotobreather solution, along with the instantaneous central plaquettes vorticity (b).

in terms of rotobreather solutions. It is important to note that both discreteness of the system and periodic couplings are prerequisites for these solutions to occur.

\section{Linear stability analysis}

For a time-periodic trajectory of a dynamical system, its linear stability can be reduced to that of the fixed point of the Poincaré map obtained from the trajectory after stroboscopic section (Floquet analysis).

Let $N$ denote the size (number of vertical links) of the ladder. To carry out the linear stability analysis of a breather (periodic) solution $\left\{\chi_{i}(t)=0, \phi_{i}(t)\right\}$, we have to integrate the linearized equations of motion around the solution,

$$
\begin{aligned}
\delta \ddot{\chi}_{i}= & J_{x}\left[\cos \left(\phi_{i+1}-\phi_{i}\right)\left(\delta \chi_{i+1}-\delta \chi_{i}\right)+\cos \left(\phi_{i-1}-\phi_{i}\right)\left(\delta \chi_{i-1}-\delta \chi_{i}\right)\right] \\
& +\epsilon_{x}\left(\delta \dot{\chi}_{i+1}+\delta \dot{\chi}_{i-1}-2 \delta \dot{\chi}_{i}\right), \\
\delta \ddot{\phi}_{i}= & J_{x}\left[\cos \left(\phi_{i+1}-\phi_{i}\right)\left(\delta \phi_{i+1}-\delta \phi_{i}\right)+\cos \left(\phi_{i-1}-\phi_{i}\right)\left(\delta \phi_{i-1}-\delta \phi_{i}\right)\right] \\
& +\epsilon_{x}\left(\delta \dot{\phi}_{i+1}+\delta \dot{\phi}_{i-1}-2 \delta \dot{\phi}_{i}\right)-2 J_{y} \cos \left(2 \phi_{i}\right) \delta \phi_{i}-2 \epsilon_{y} \delta \dot{\phi}_{i},
\end{aligned}
$$


over a period $T_{\mathrm{b}}$, for each of the $4 N$-initial conditions of a vector basis of the $4 N$-dimensional vector space formed by the variables $\left\{\delta \chi_{i}, \delta \phi_{i}, \delta \dot{\chi}_{i}, \delta \dot{\phi}_{i}\right\}$. With this calculation we obtain the monodromy (or Floquet) matrix of the rotobreather,

$$
\left(\delta \chi_{i}, \delta \phi_{i}, \delta \dot{\chi}_{i}, \delta \dot{\phi}_{i}\right)_{t=T_{\mathrm{b}}}^{\mathrm{T}}=\mathcal{M}\left(\delta \chi_{i}, \delta \phi_{i}, \delta \dot{\chi}_{i}, \delta \dot{\phi}_{i}\right)_{t=0}^{\mathrm{T}},
$$

where $(\delta \chi, \ldots)^{\mathrm{T}}$ denotes column vector. The eigenvalues of the monodromy matrix are the Floquet multipliers. They contain all the information about the linear stability of the periodic solution. Linear stability is assured when all the eigenvalues lie inside the unit circle. Note that, since the system is real, for each eigenvalue $v$ its complex conjugate $\nu^{*}$ is also an eigenvalue. Note also that the freedom in the choice of the values of $\Omega$ and $\alpha$ for the $\chi$-components of the breather solution implies that two eigenvalues at +1 will always be present in the spectrum of $\mathcal{M}$. The corresponding eigenvectors are extended modes, uniform in the $\delta \dot{\chi}$ and $\delta \chi$ components, respectively (and zero for the $\delta \dot{\phi}$ and $\delta \phi$ components).

In our numerical investigations of linear stability of rotobreathers solutions in the anisotropic Josephson junction ladder, we have up to now kept fixed the value of the parameters $J_{y}=0.5, \epsilon_{y}=0.01$ and $\omega=1.5$, while exploring in the three-dimensional parameter space $\left(J_{x}, \epsilon_{x}, I_{\mathrm{AC}}\right)$.

\section{1. $\chi$-Instability bifurcation}

The first type of instability that we describe here is generally found when the Josephson coupling in the horizontal direction increases above values around $J_{x} \simeq 0.105$. As the value of $J_{x}$ increases, two complex conjugate eigenvalues are seen to collide on the negative side of the real axis, in the interior of the unit circle, then separate apart moving in opposite directions along the real axis, so that one of them leaves the unit circle at -1 . The associated eigenvector has zero components along $\delta \phi_{i}$ and $\delta \dot{\phi}_{i}$, and non-zero components along $\delta \chi_{i}$ and $\delta \dot{\chi}_{i}$ exponentially localized around $j^{*}$, the center of the rotobreather solution. The localization length scale (width) of this eigenvector is much larger than that of the rotobreather, by more than an order of magnitude, typically. This is likely due to the absence of on-site pinning potential term in the equations of motion (1) for the $\chi$ variables.

Fig. 2 shows a snapshot of the periodic solution obtained by integration of the full equations of motion (1) and (2), with initial conditions prepared by slightly perturbing the rotobreather along the direction of the destabilizing eigenvector, for the parameter values $\epsilon_{x}=0.01, I_{\mathrm{AC}}=0.3$, and $J_{x}=0.108$ (just above the bifurcation). As seen in the figure, the periodic solution obtained can be described as a rotobreather in the $\phi_{i}$ variables (almost unchanged from the rotobreather before the bifurcation) and an oscillating localized mode in the $\chi_{i}$ variables. This $\chi$-breather has a period twice the one of the rotobreather before bifurcation, is spatially symmetric and exhibits an "antiferro-like" profile, like the destabilizing eigenvector driving the bifurcation.

The value of the Josephson horizontal coupling $J_{x}$ where this $\chi$-instability bifurcation occurs is observed to be almost insensitive to changes in the parameters $I_{\mathrm{AC}}$ and $\epsilon_{x}$. In order to get some insight into the mechanism of this bifurcation, and based on this last observation, let us consider the linearized equations (3) and drop out the "phonon damping" term in their right-hand side:

$$
\delta \ddot{\chi}_{i}=J_{x}\left[\cos \left(\phi_{i+1}-\phi_{i}\right)\left(\delta \chi_{i+1}-\delta \chi_{i}\right)+\cos \left(\phi_{i-1}-\phi_{i}\right)\left(\delta \chi_{i-1}-\delta \chi_{i}\right)\right] .
$$

These equations can be reinterpreted as the equations of motion of an acoustic chain of harmonic oscillators, with couplings $C_{i}=J_{x} \cos \left(\phi_{i+1}(t)-\phi_{i}(t)\right)$ which are periodic functions in time. This system is expected to be stable for small values of the coupling parameter $J_{x}$, and develops a parametric (subharmonic) instability when $J_{x}$ becomes large enough, in analogy with the well-known single parametric oscillator (Mathieu-Hill equation). Since the coefficients $C_{i}$ become time-independent and uniform for large values of $\left|i-j^{*}\right|$, the instability of the chain necessarily occurs by a localized mode. 

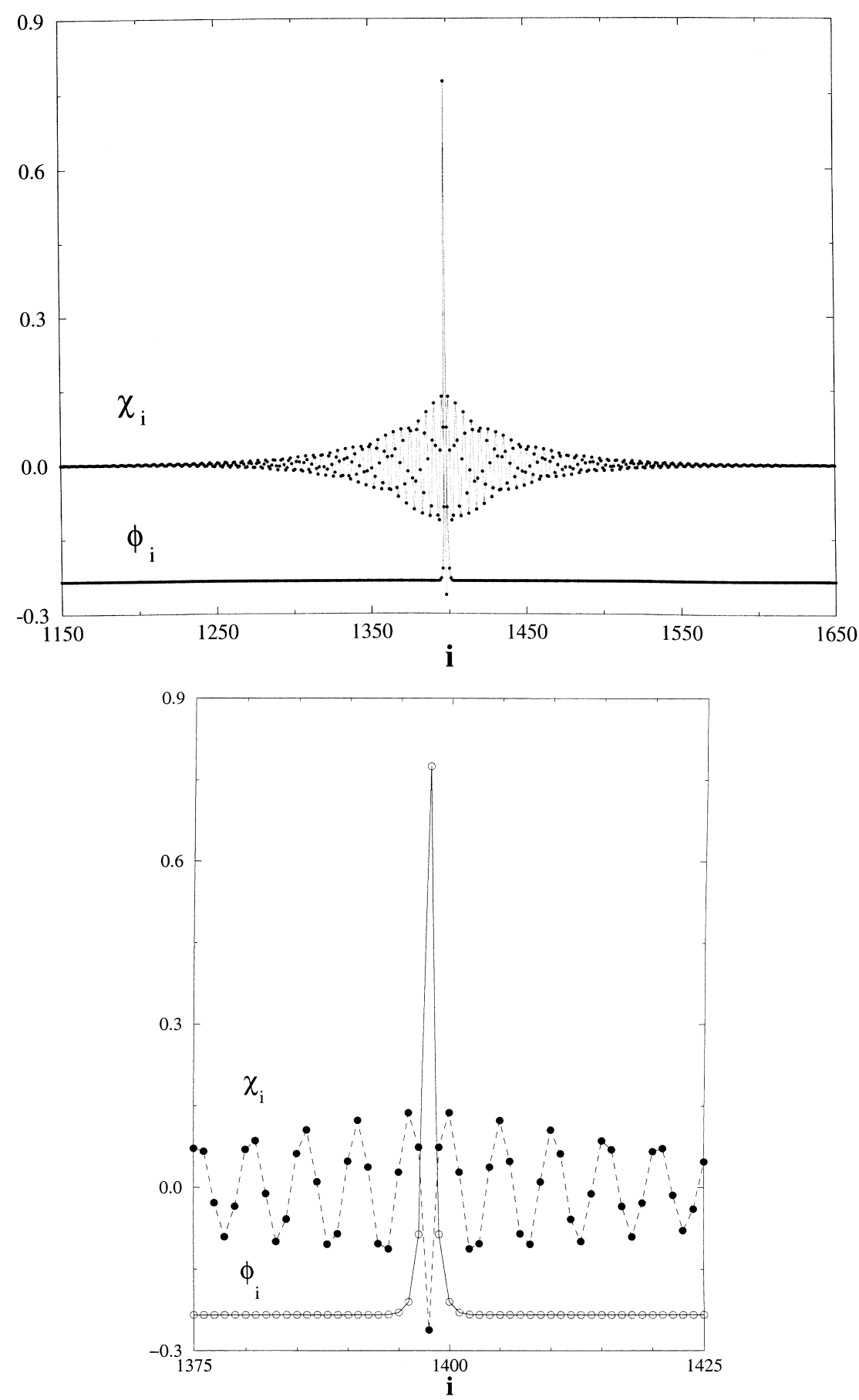

Fig. 2. Snapshot of the profiles of $\phi_{i}$ and $\chi_{i}$ for the attracting rotobreather solution above the $\chi$-instability bifurcation $\left(\epsilon_{x}=0.01\right.$, $I_{\mathrm{AC}}=0.3$, and $J_{x}=0.108$ ). The central rotating variable $\phi_{j} *$ is drawn modulo $2 \pi$. Note the different localization width scales for both profiles. 

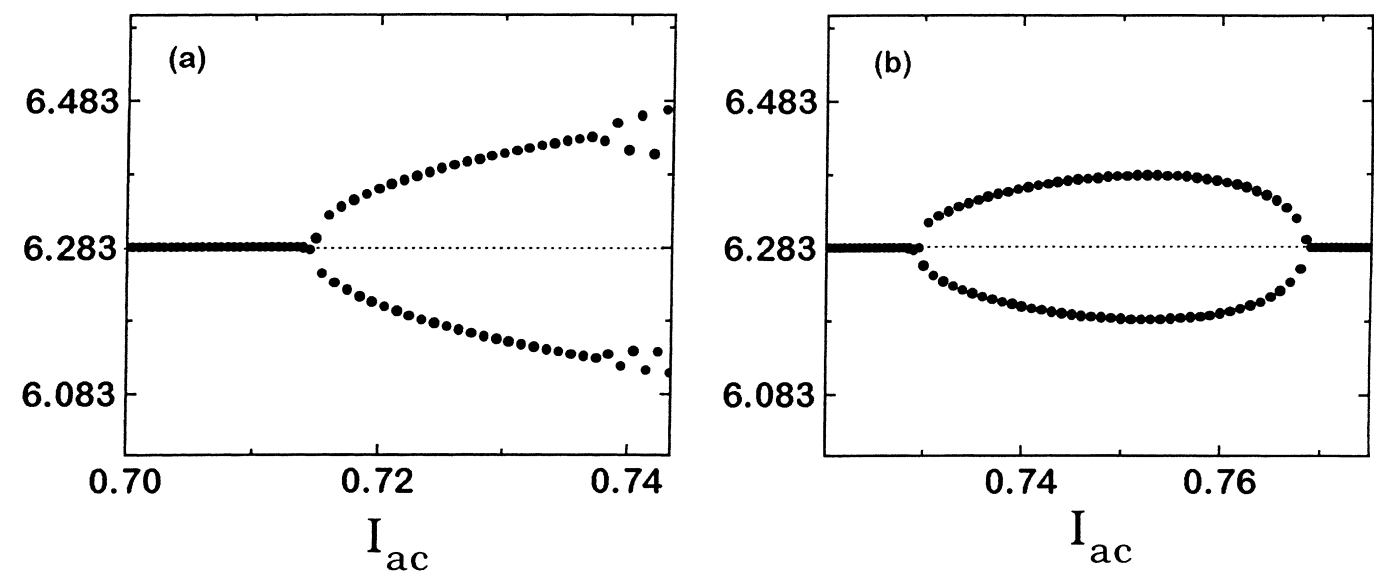

Fig. 3. Period-doubling bifurcation. We plot here $\phi_{j^{*}}\left(t+T_{b}\right)-\phi_{j^{*}}(t)$ as a function of $I_{\mathrm{AC}}$, where $j^{*}$ is the the central site of the rotobreather. (a) For $\epsilon_{x}=0.0275$ and $J_{x}=0.05$; a second period-doubling is observed. (b) For $\epsilon_{x}=0.0275$ and $J_{x}=0.055$, an inverse period-doubling occurs after increase of $I_{\mathrm{AC}}$.

The linear stability analysis of the attracting periodic solution ( $\chi$-breather plus $\phi$-rotobreather) above the bifurcation is hard to perform properly, due to the large size of the system needed to allocate the width of the $\chi$-breather (which in fact increases with $J_{x}$ ). Our numerical computations with sizes up to $N=3 \times 10^{3}$ indicate that it becomes unstable shortly after $J_{x}$ is increased further by a very small amount, of the order of $\Delta J_{x} \simeq 0.01$.

\subsection{Period-doubling bifurcation}

A second type of instability is found when the currents intensity $I_{\mathrm{AC}}$ is increased, while keeping fixed $J_{x}$ and $\epsilon_{x}$. Like in the bifurcation previously analyzed, two complex conjugate eigenvalues collide inside the unit disk, on the real axis close to -1 , then separate apart along opposite directions on the real axis, one of them eventually reaching -1 . The associated eigenvector has now zero components along $\delta \chi_{i}$ and $\delta \dot{\chi}_{i}$, and non-zero components along $\delta \phi_{i}$ and $\delta \dot{\phi}_{i}$ which are exponentially localized around $i=j^{*}$, the center of the rotobreather. The localization width of this eigenvector is about the same as the one for the rotobreather solution.

When initial conditions are prepared by slightly perturbing the rotobreather along the direction of the destabilizing eigenvector, a rotobreather solution with period double than the one before bifurcation is reached. In Fig. 3 we show $\phi_{j^{*}}\left(t+T_{b}\right)-\phi_{j^{*}}(t)$ for the new breather solution as a function of $I_{\mathrm{AC}}$. The bifurcation is thus a typical perioddoubling bifurcation. Sometimes, as shown in Fig. 3(a), further period-doubling bifurcations occur, though we have not found any clear example of a complete subharmonic cascade. Further increase of $I_{\mathrm{AC}}$ usually leads to the destruction of the rotobreather, which falls into the basin of attraction of the uniformly oscillating solution, often before a second period-doubling bifurcation could take place. Interestingly, for some ranges of values of $J_{x}$ and $\epsilon_{x}$, when the current intensity $I_{\mathrm{AC}}$ is increased beyond the first period-doubling bifurcation, an inverse period-doubling occurs, which brings the rotobreather solution back to the previous period (see Fig. 3(b)). In this case, further increase of $I_{\mathrm{AC}}$ ends in a bifurcation of the type which we will analyze in the next Section.

\subsection{Dangerous bifurcation}

The third type of bifurcation destabilizing the rotobreather is generally found when the parameter $\epsilon_{x}$ is increased. As shown in Fig. 4, two complex conjugate eigenvalues collide inside the unit disk, on the positive part of the real 


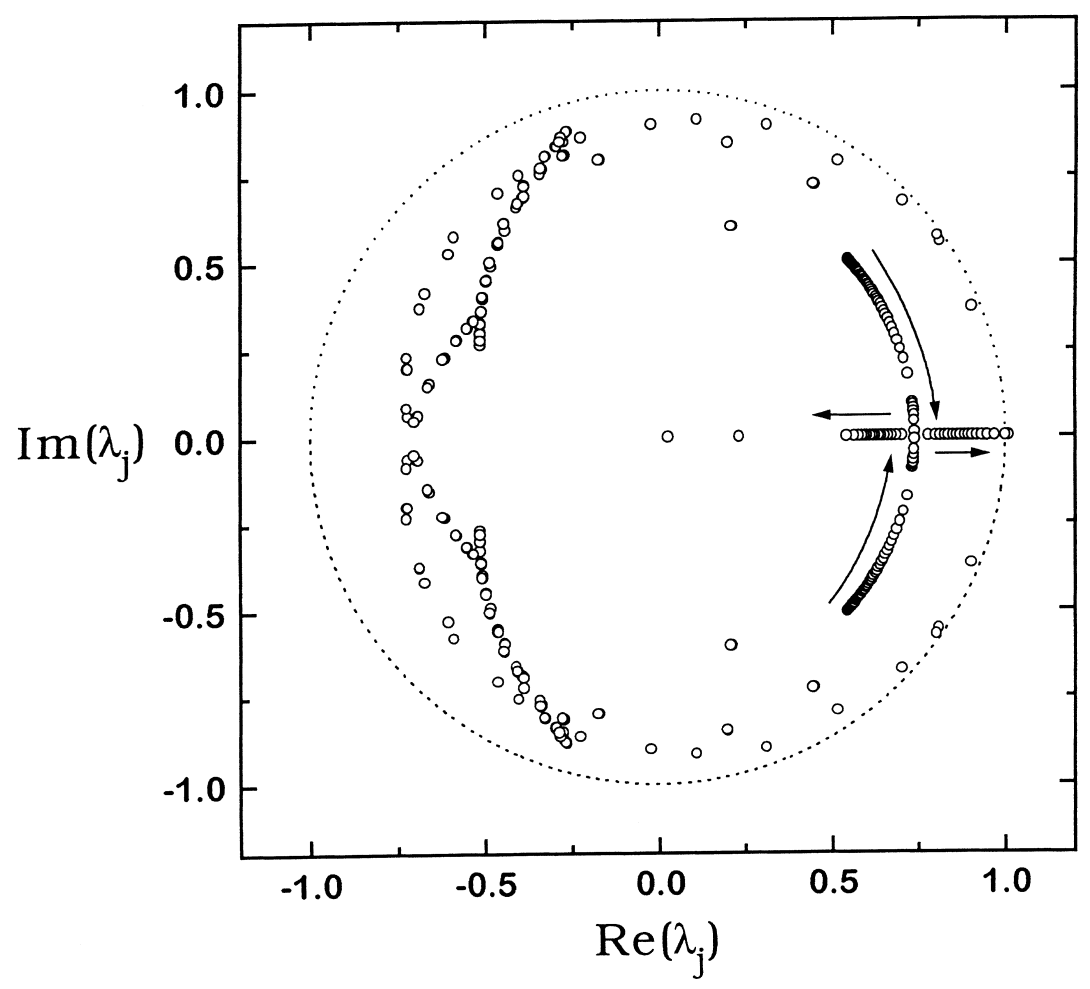

Fig. 4. Dangerous bifurcation. The Floquet multipliers $\lambda_{\mathrm{j}}$, for values of $\epsilon_{x}$ in the interval [0.0279, 0.0279822], are drawn in the complex plane $\left(J_{x}=0.05, I_{\mathrm{AC}}=0.3\right)$. Only one colliding pair of eigenvalues varies significantly in this $\epsilon_{x}$-interval.

axis, then separate apart in opposite directions along the real axis, so that one of them eventually leaves the unit disk at +1 . The eigenvector has zero components along $\delta \chi_{i}$ and $\delta \dot{\chi}_{i}$, and non-zero components along $\delta \phi_{i}$ and $\delta \dot{\phi}_{i}$ which are exponentially localized around $i=j^{*}$, with localization width of the order of that of the rotobreather.

Perturbation of the rotobreather solution along the destabilizing eigenvector brings it into the basin of attraction of the uniformly oscillating solution. According to some recently proposed classification of generic codimension1 attractor bifurcations of dissipative dynamical systems [15], this one would fit the description of dangerous bifurcations, which are characterized by "the blue sky disappearance of the current attractor, giving rise to a jump to a remote attractor of any type". In our case it is likely a saddle-node bifurcation, though this issue deserves further confirmation. The bifurcation value of $\epsilon_{x}$ increases with increasing current intensity $I_{\mathrm{AC}}$, a fact which agrees with the simple physical intuition that the effects of both parameters on the central rotating $\phi_{j^{*}}(t)$ oppose each other.

\section{Concluding remarks}

In Fig. 5 we show some two-dimensional sections of the stability phase diagram of the family of rotobreathers of period $T_{\mathrm{b}}$ in the three-dimensional parameter space $\left(J_{x}, \epsilon_{x}, I_{\mathrm{AC}}\right)$, for fixed values of the parameters $J_{y}=0.5$, $\epsilon_{y}=0.01$ and $\omega=1.5$. For the sake of clarity, we have not included there the bifurcation lines corresponding to second, third, etc. period-doubling transitions which, as mentioned, have been observed to occur in some regions. 

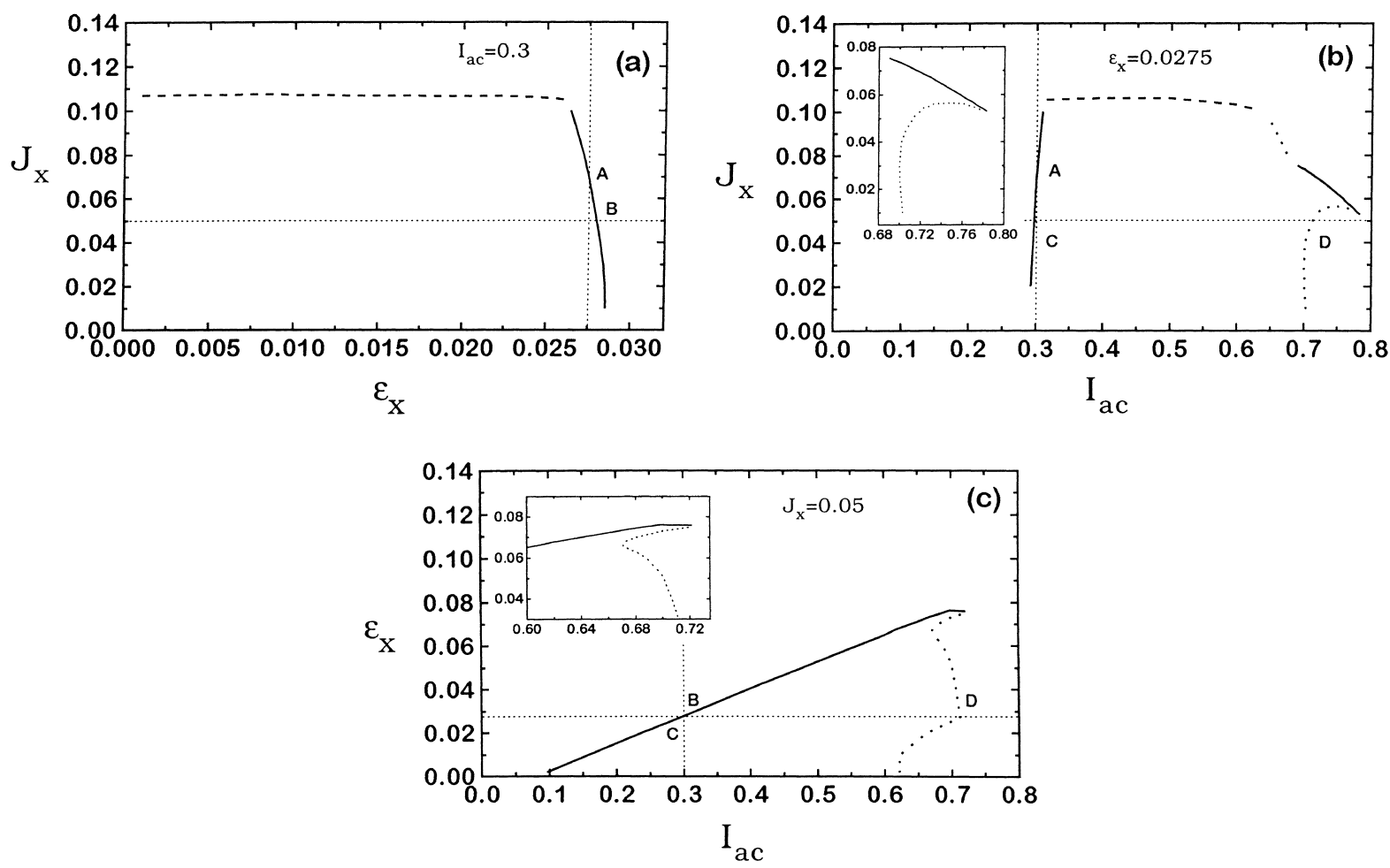

Fig. 5. Two-dimensional sections of the stability phase diagram. Widely dotted lines correspond to period doubling bifurcations, broken lines represent $\chi$-instability bifurcations, and continuous lines indicate dangerous bifurcations. Some points $(A, B, C$ and $D)$ which are common to a pair of planes are indicated, to help $3 \mathrm{~d}$ mental reconstruction of the diagram. Tightly-dotted straight lines in each plane are the corresponding intersections with the other planes. The insets show enlargements of the corresponding areas.

Besides the three types of bifurcation we have analyzed here ( $\chi$-instability, period doubling and dangerous), which are always mediated by localized eigenmodes, we have observed instabilities driven by extended eigenmodes. However, these were not true extended instabilities of the full lattice, which in principle are possible for the $\phi$-system of Eqs. (2). They were found to be finite size effects, similar to the ones encountered generically for breathers in Hamiltonian lattices. In such systems it has been conjectured [14], on the basis of heuristic arguments, that extended instabilities necessarily become marginal in the limit of the infinite lattice. The $\chi$-system of equations (2) can be shown to exhibit this behavior too, since its associated linearized problem for the reference breather solution $\left\{\phi_{i}^{b}(t), \chi_{i}=0\right\}$ yields a lattice with a parametric forcing that vanishes as we go away from the center $i=j^{*}$. That is, no extended instabilities are allowed in the $\chi$-system in this case, and the numerical results confirm this: extended instabilities were observed near the $\chi$-instability, but they vanished as the size $N$ of the lattice was progressively increased, leaving the localized (though broad), unstable mode out in the clear. In the view of the broad width of this mode, it is not surprising to have hit these finite size effects.

Further work on linear stability of rotobreather solutions in anisotropic Josephson junction ladders has to be done, by varying parameters like the frequency of the external currents and vertical junction characteristics. Also, oscillating breather solutions have to be considered in this respect. Finally, one should consider the role of a small DC current superimposed to the AC current in causing possibly different types of instabilities. These are only a very small part of the issues that remain open concerning the general problem of nonlinear localization in Josephson junction devices. 


\section{Acknowledgements}

We would like to thank C. Baesens, A.R. Bishop, F. Falo, S. Flach, R.S. Mackay, M. Salerno and J.-A. Sepulchre for interesting discussions, and H.S.J. van der Zant for calling Ref. [13] to our attention. We acknowledge support from DGES (PB95-0797), Spain. One of us (JJM) acknowledges a Fullbright-MEC fellowship.

\section{References}

[1] L.M. Floría, J.L. Marín, P.J. Martínez, F. Falo, S. Aubry, Europhys. Lett. 36 (1996) 539.

[2] L.M. Floría, J.L. Marín, S. Aubry, P.J. Martínez, F. Falo, J.J. Mazo, Physica D 113 (1998) 387.

[3] M. Tinkham, Introduction to Superconductivity, 2nd ed., McGraw-Hill, New York, 1996.

[4] S. Flach, C.R. Willis, Discrete breathers, Phys. Rep. 295 (1998) 181.

[5] S. Aubry, S. Flach, K. Kladko, E. Olbrich, Phys. Rev. Lett. 76 (1996) 1607.

[6] S. Watanabe, H.S.J. van der Zant, S.H. Strogatz , T. Orlando, Physica D 97 (1996) 429.

[7] R.S. Mackay, S. Aubry, Nonlinearity 7 (1994) 1623.

[8] J.L. Marín, S. Aubry, Nonlinearity 9 (1996) 1501.

[9] R.S. Mackay, J.-A. Sepulchre, Physica D 119 (1998) 148.

[10] S. Takeno, M. Peyrard, Physica D 92 (1996) 140.

[11] S. Takeno, M. Peyrard, Nonlinear rotating modes: Green's function solution, Preprint, 1996.

[12] S. Aubry, Physica D 103 (1997) 201.

[13] W.J. Elion, J.J. Wachters, L.L. Sohn, J.E. Mooij, Phys. Rev. Lett. 71 (1993) 2311

[14] J.L. Marín, S. Aubry, Physica D 119 (1998) 163; J.L. Marín, Ph.D. dissertation, Universidad de Zaragoza, 1997 (available upon request to author).

[15] J.M.T. Thompson, H.B. Stewart, Y. Ueda, Phys. Rev. E 49 (1994) 1019. 